\title{
Analysis of radial vibrations of poroelastic circular cylindrical shells immersed in an acoustic medium
}

\author{
M. Ramesh$^{1}$, S. Ahmed Shah ${ }^{2}$, M.V. Ramana Murthy \\ ${ }^{I}$ Department of Mathematics, University College of Technology, Osmania University, Hyderabad, INDIA \\ ${ }^{2 *}$ Department of Mathematics, Deccan College of Engineering and Technology, Hyderabad, INDIA \\ ${ }^{3}$ Department of Mathematics, Osmania University, Hyderabad, INDIA \\ "Corresponding Author: e-mail: ahmed_shah67@yahoo.com
}

\begin{abstract}
Waves propagating in radial direction of a poroelastic circular cylinder are termed as radial vibrations. Radial vibrations of poroelastic circular cylindrical shell of infinite extent immersed in an inviscid elastic fluid are examined employing Biot's theory. Biot's model consists of an elastic matrix permeated by a network of interconnected spaces saturated with liquid. Frequency equation is obtained each for a permeable and an impermeable surface. Poroelastic cylindrical shell is assumed to be homogeneous and isotropic. Particular cases are considered when the outer and inner fluid vanishes. (1). When the outer fluid is vanished, the considered problem reduces to the problem of radial vibrations of fluid-filled poroelastic circular cylindrical shell. (2). When the inner fluid vanishes, the considered problem reduces to the problem of radial vibrations of poroelastic circular cylindrical shell surrounded by fluid. (3). When both outer and inner fluids vanish, the considered problem is reduced to the problem of radial vibrations of poroelastic circular cylindrical shell in vacuum. For the case, when the inner radius of the poroelastic cylindrical shell approaches to zero, the considered problem is reduced to radial vibrations of poroelastic solid cylinder immersed in fluid. Frequency equation each for a permeable and an impermeable surface is obtained for the said cases. Effect of wall-thickness of the poroelastic circular cylindrical shell in presence of fluid is studied for different cases. Results of previous investigations are shown as a particular case of the present study. By ignoring the liquid effects, the results of purely elastic solid are obtained as a special case.
\end{abstract}

Keywords: Radial vibrations, cylindrical shell, frequency, elastic fluid, permeable surface, impermeable surface.

DOI: http://dx.doi.org/10.4314/ijest.v6i5.3

\section{Introduction}

The study of propagation of waves is important in different fields of engineering and geophysics, particularly in the area of destructive research. McFadden (1954) studied the radial vibrations of thick-walled hollow elastic cylinders of infinite extent. Gazis (1959) discussed the propagation of free harmonic waves along a hollow elastic circular cylinder of infinite extent and solved the frequency equation using bisection method. Employing Biot's (1956) theory, Wisse et al (2002) presented the experimental results of guided wave modes in porous cylinders. Chao et al (2004) studied the shock-induced borehole waves in porous formations. Wave propagation in fluid-loaded transversely isotopic cylinders is studied by Marilyn and Solecki (1996). Ahmed Shah $(2008,2011)$ investigated the axially symmetric vibrations of fluid-filled poroelastic cylindrical shells and flexural vibrations of coated poroelastic cylinders in absence of dissipation. Ahmed Shah (2012) studied axially symmetric vibrations of poroelastic cylindrical shell immersed in fluid and showed that the radial and shear vibrations are uncoupled when the wavenumber is vanished. The phase velocity is obtained and presented graphically for different thicknesses of the poroelastic shell. Tajuddin and Ahmed Shah (2010) studied radial vibrations of hollow poroelastic cylinders. The frequencies of radial vibrations are obtained for thin shell as a limiting case. Ahmed Shah and Tajuddin $(2009,10,11)$ investigated different problems characterizing Biot's theory. In the paper Ahmed Shah and Tajuddin (2010) flexural wave propagation in poroelastic cylindrical 
shell immersed in an acoustic medium is considered. They showed that the phase velocity of an impervious surface is higher than that of a pervious surface in case of kerosene saturated sandstone while the frequency of a pervious surface is higher than that of an impervious surface for kerosene saturated sandstone and water saturated sandstone. Wave propagation in composite hollow sphere and fluid-filled sphere surrounded by fluid are analyzed by Shanker et. al (2013a, 2013b) wherein they obtained the frequency equations for rigid casing and rigid core for a pervious and an impervious surface.

In the present analysis, the radial vibrations of poroelastic circular cylindrical shells of infinite extent immersed in an acoustic medium are investigated employing Biot's (1956) theory. Biot's model consists of an elastic matrix permeated by a network of interconnected spaces saturated with liquid. The frequency equation of radial vibrations of poroelastic cylindrical shells immersed in an inviscid elastic fluid is derived each for a permeable surface and an impermeable surface. Non-dimensional frequency for propagating modes is computed in absence of dissipation for poroelastic cylindrical shells immersed in an elastic fluid each for a permeable and an impermeable surface. The results are presented graphically for two types of poroelastic materials and then discussed. Results of some previous works are shown as a special case of the present investigation. By ignoring liquid effects and after some rearrangement of terms, the results of purely elastic solid are obtained.

\section{Governing equations}

The equations of motion of a homogeneous, isotropic poroelastic solid (Biot, 1956) in presence of dissipation $b$ are

$$
\begin{array}{r}
N \nabla^{2} \vec{u}+(A+N) \nabla e+Q \nabla \in=\frac{\partial^{2}}{\partial t^{2}}\left(\rho_{11} \vec{u}+\rho_{12} \vec{U}\right)+b \frac{\partial}{\partial t}(\vec{u}-\vec{U}), \\
Q \nabla e+R \nabla \in=\frac{\partial^{2}}{\partial t^{2}}\left(\rho_{12} \vec{u}+\rho_{22} \vec{U}\right)-b \frac{\partial}{\partial t}(\vec{u}-\vec{U}),
\end{array}
$$

where $\nabla^{2}$ is the Laplacian, $\overrightarrow{\mathrm{u}}=(u, v, w)$ and $\overrightarrow{\mathrm{U}}=(U, V, W)$ are displacements of solid and liquid media, respectively; e and $\in$ are the dilatations of solid and liquid; $A, N, Q, R$ are all poroelastic constants and $\rho_{i j} \quad(i, j=1,2)$ are the mass coefficients following Biot (1956). The poroelastic constants $A$ and $N$ correspond to familiar Lame constants in purely elastic solid. The coefficient $N$ represents the shear modulus of the solid. The coefficient $R$ is a measure of the pressure required on the liquid to force a certain amount of the liquid into the aggregate while total volume remains constant. The coefficient $Q$ represents the coupling between the volume change of the solid to that of liquid.

The stresses $\sigma_{\mathrm{ij}}$ and the liquid pressure $\mathrm{s}$ of the poroelastic solid are

$$
\begin{aligned}
\sigma_{i j} & =2 N e_{i j}+(A e+Q \in) \delta_{i j}, \quad(i, j=r, \theta, z), \\
s & =Q e+R \in,
\end{aligned}
$$

where $\delta_{\mathrm{ij}}$ is the well-known Kronecker delta function.

The equation of motion for a homogeneous, isotropic, inviscid elastic fluid is

$$
\nabla^{2} \Phi=\frac{1}{V_{f}^{2}} \frac{\partial^{2} \Phi}{\partial t^{2}},
$$

where $\Phi$ is displacement potential function and $V_{\mathrm{f}}$ is the velocity of sound in the fluid.

The displacement of fluid is $\overrightarrow{\mathrm{u}}_{\mathrm{f}}=\left(u_{\mathrm{f}}, v_{\mathrm{f}}, w_{\mathrm{f}}\right)$.

The fluid pressure $P_{\mathrm{f}}$ is given by

$$
P_{f}=-\rho_{f} \frac{\partial^{2} \Phi}{\partial t^{2}}
$$

In equation (4), $\rho_{\mathrm{f}}$ is the density of the fluid.

Hereafter the subscript 'if' or 'of' is associated with a fluid quantity represents that the said quantity relates to inner or outer fluid. For example, $V_{\text {if }}$ is the velocity of sound in the inner fluid and $P_{\text {of }}$ is the outer fluid pressure.

\section{Solution of the problem}

Let $(r, \theta, z)$ be the cylindrical polar coordinates. Consider a homogeneous, isotropic, infinite poroelastic cylindrical shell immersed in an inviscid elastic fluid. Let the inner and outer radii of poroelastic cylindrical shell be $r_{1}$ and $r_{2}$, respectively, so that the wall-thickness of the shell is $\mathrm{h}\left[=\left(\mathrm{r}_{2}-\mathrm{r}_{1}\right)>0\right]$. The axis of the poroelastic shell is in the direction of $\mathrm{z}$-axis. 


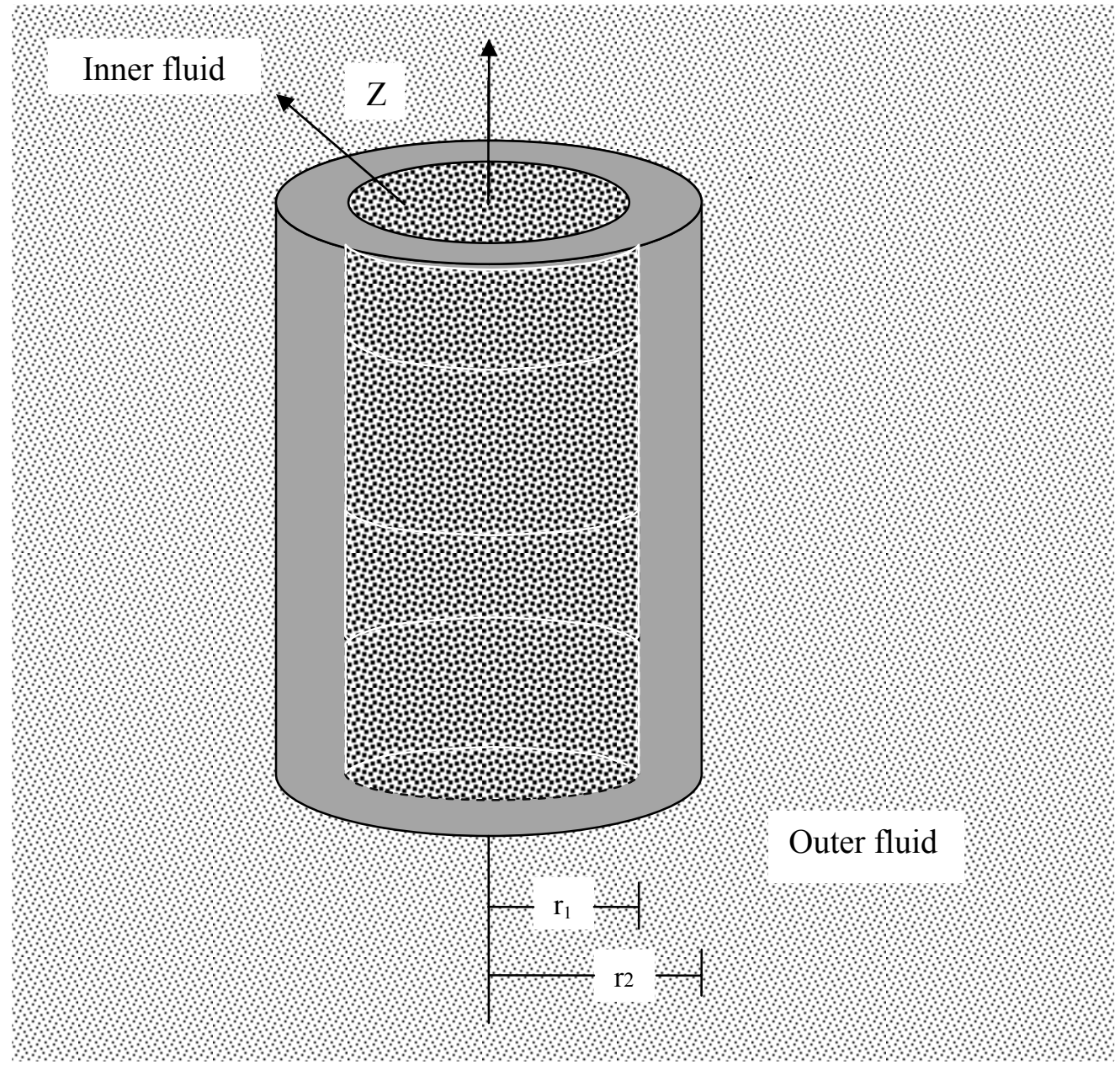

The fluid column within the poroelastic cylindrical shell extends from zero to infinity in axial direction and zero to $r_{1}$ in the radial direction. The outer fluid extends from $\mathrm{r}_{2}$ to infinity in radial direction and zero to infinity in axial direction. The only non-zero displacement component of radial vibrations both for solid and liquid media are $\overrightarrow{\mathrm{u}}(u, 0,0), \overrightarrow{\mathrm{U}}(U, 0,0)$, respectively.

These displacements are functions of $r$ and time, t. The solid and liquid displacement functions which can readily be evaluated for steady state harmonic vibrations from equations (1) are

$$
\begin{aligned}
u(r, t) & =\left[C_{1} J_{1}\left(\xi_{1} r\right)+C_{2} Y_{1}\left(\xi_{1} r\right)+C_{3} J_{1}\left(\xi_{2} r\right)+C_{4} Y_{1}\left(\xi_{2} r\right)\right] \exp (i \omega t), \\
U(r, t) & =-\left[C_{1} \delta_{1}^{2} J_{1}\left(\xi_{1} r\right)+C_{2} \delta_{1}^{2} Y_{1}\left(\xi_{1} r\right)+C_{3} \delta_{2}^{2} J_{1}\left(\xi_{2} r\right)+C_{4} \delta_{2}^{2} Y_{1}\left(\xi_{2} r\right)\right] \exp (i \omega t),
\end{aligned}
$$

where $\omega$ is the frequency of the wave, $\mathrm{C}_{1}, \mathrm{C}_{2}, \mathrm{C}_{3}, \mathrm{C}_{4}$ are all constants, $J_{\mathrm{n}}$ and $Y_{\mathrm{n}}$ are Bessel functions of the first and second kind of order $n$ and $\delta_{\mathrm{i}}^{2}, \xi_{\mathrm{i}}(\mathrm{i}=1,2)$ are

$$
\delta_{i}^{2}=\frac{1}{\left(R K_{12}-Q K_{22}\right)}\left[\left(R K_{11}-Q K_{12}\right)-V_{i}^{-2}\left(P R-Q^{2}\right)\right], \quad \xi_{i}=\frac{\omega}{V_{i}}, \quad P=A+2 N .
$$

In equation (6), $V_{1}$ and $V_{2}$ are the dilatational wave velocities of first and second kind, respectively and $K_{11}, K_{12}, K_{22}$ are

$$
K_{11}=\rho_{11}-\frac{i b}{\omega}, \quad K_{12}=\rho_{12}+\frac{i b}{\omega}, \quad K_{22}=\rho_{22}-\frac{i b}{\omega} .
$$

The dilatations of solid and liquid media following Biot (1956) are

$$
e=\left[f^{\prime}+\frac{1}{r} f\right] \exp (i \omega t), \quad \in=\left[F^{\prime}+\frac{1}{r} F\right] \exp (i \omega t),
$$

where a 'dash' over a quantity denote differentiation with respect to $r$.

The displacement of inner fluid column $\overrightarrow{\mathrm{u}}_{\mathrm{if}}\left[=\left(u_{\mathrm{if}}, 0,0\right)\right]$ for radial vibrations is 
where $A_{\text {if }}$ is constant and $\xi_{\text {if }}^{2}$ are

$$
u_{i f}=A_{i f} J_{1}\left(\xi_{i f} r\right) \exp (i \omega t), \quad v_{i f}=0, \quad w_{i f}=0,
$$

$$
\xi_{i f}^{2}=\frac{\omega^{2}}{V_{i f}^{2}} .
$$

With the help of displacement potential function, inner fluid pressure $P_{\text {if }}$ is

$$
P_{i f}=A_{i f} \omega^{2} \rho_{i f} J_{1}\left(\xi_{i f} r\right) \exp (i \omega t) .
$$

Similarly, the displacement of the outer fluid and the outer fluid pressure are given by the equations

$$
u_{o f}=A_{o f} H_{1}^{(2)}\left(\xi_{o f} r\right) \exp (i \omega t), \quad v_{o f}=0, \quad w_{o f}=0, \quad P_{o f}=A_{o f} \omega^{2} \rho_{o f} H_{1}^{(2)}\left(\xi_{o f} r\right) \exp (i \omega t),
$$

where $A_{\mathrm{of}}$ is constant, $H_{\mathrm{n}}{ }^{(2)}$ is Hankel function of second kind of order $\mathrm{n}$ and $\xi_{\mathrm{of}}{ }^{2}$ is

$$
\xi_{o f}^{2}=\frac{\omega^{2}}{V_{o f}^{2}} \text {. }
$$

Substituting the displacement function $u$ from equation (5), fluid pressures from equations (11) and (12) into equations (2) and (4), the relevant displacement, liquid pressure and stresses are

$$
\begin{aligned}
& s=\left[C_{1} M_{21}(r)+C_{2} M_{22}(r)+C_{3} M_{23}(r)+C_{4} M_{24}(r)\right] \exp (i \omega t), \\
& u-u_{i f}=\left[C_{1} M_{31}(r)+C_{2} M_{32}(r)+C_{3} M_{33}(r)+C_{4} M_{34}(r)+A_{i f} M_{35}(r)\right] \exp (i \omega t), \\
& \sigma_{r r}+s+P_{o f}=\left[C_{1} M_{41}(r)+C_{2} M_{42}(r)+C_{3} M_{43}(r)+C_{4} M_{44}(r)+A_{o f} M_{46}(r)\right] \exp (i \omega t), \\
& u-u_{o f}=\left[C_{1} M_{61}(r)+C_{2} M_{62}(r)+C_{3} M_{63}(r)+C_{4} M_{64}(r)+A_{o f} M_{66}(r)\right] \exp (i \omega t), \\
& \frac{\partial s}{\partial r}=\left[C_{1} N_{21}(r)+C_{2} N_{22}(r)+C_{3} N_{23}(r)+C_{4} N_{24}(r)\right] \exp (i \omega t),
\end{aligned}
$$

where the elements $\mathrm{M}_{\mathrm{ij}}(\mathrm{r})$ and $\mathrm{N}_{\mathrm{ij}}(\mathrm{r})$ are

$$
\begin{aligned}
& M_{11}(r)=\frac{-2}{r}\left[(Q+R) \delta_{1}^{2}-(P+Q-N)\right] J_{1}\left(\xi_{1} r\right)+\left[(Q+R) \delta_{1}^{2}-(P+Q)\right] \xi_{1} J_{2}\left(\xi_{1} r\right), \\
& M_{12}(r)=\frac{-2}{r}\left[(Q+R) \delta_{1}^{2}-(P+Q-N)\right] Y_{1}\left(\xi_{1} r\right)+\left[(Q+R) \delta_{1}^{2}-(P+Q)\right] \xi_{1} Y_{2}\left(\xi_{1} r\right), \\
& M_{13}(r)=\frac{-2}{r}\left[(Q+R) \delta_{2}^{2}-(P+Q-N)\right] J_{1}\left(\xi_{2} r\right)+\left[(Q+R) \delta_{2}^{2}-(P+Q)\right] \xi_{2} J_{2}\left(\xi_{2} r\right), \\
& M_{14}(r)=\frac{-2}{r}\left[(Q+R) \delta_{2}^{2}-(P+Q-N)\right] Y_{1}\left(\xi_{2} r\right)+\left[(Q+R) \delta_{2}^{2}-(P+Q)\right] \xi_{2} Y_{2}\left(\xi_{2} r\right), \\
& M_{15}(r)=\omega^{2} \rho_{i f} J_{1}\left(\xi_{i f} r\right), \quad M_{16}(r)=0, \\
& M_{21}(r)=\frac{-2}{r}\left(R \delta_{1}^{2}-Q\right) J_{1}\left(\xi_{1} r\right)+\left(R \delta_{1}^{2}-Q\right) \xi_{1} J_{2}\left(\xi_{1} r\right) \\
& M_{22}(r)=\frac{-2}{r}\left(R \delta_{1}^{2}-Q\right) Y_{1}\left(\xi_{1} r\right)+\left(R \delta_{1}^{2}-Q\right) \xi_{1} Y_{2}\left(\xi_{1} r\right) \text {, } \\
& M_{23}(r)=\frac{-2}{r}\left(R \delta_{2}^{2}-Q\right) J_{1}\left(\xi_{2} r\right)+\left(R \delta_{2}^{2}-Q\right) \xi_{2} J_{2}\left(\xi_{2} r\right), \\
& M_{24}(r)=\frac{-2}{r}\left(R \delta_{2}^{2}-Q\right) Y_{1}\left(\xi_{2} r\right)+\left(R \delta_{2}^{2}-Q\right) \xi_{2} Y_{2}\left(\xi_{2} r\right), \quad M_{25}(r)=0, \quad M_{26}(r)=0, \\
& M_{31}(r)=J_{1}\left(\xi_{1} r\right), \quad M_{32}(r)=Y_{1}\left(\xi_{1} r\right), \quad M_{33}(r)=J_{1}\left(\xi_{2} r\right), \\
& M_{34}(r)=Y_{1}\left(\xi_{2} r\right), \quad M_{35}(r)=-J_{1}\left(\xi_{i f} r\right), \quad M_{36}(r)=0, \\
& M_{41}(r)=M_{11}(r), \quad M_{42}(r)=M_{12}(r), \quad M_{43}(r)=M_{13}(r), \\
& M_{44}(r)=M_{14}(r), \quad M_{45}(r)=0, \quad M_{46}(r)=\omega^{2} \rho_{o f} H_{1}^{(2)}\left(\xi_{o f} r\right), \\
& M_{5 j}(r)=M_{2 j}(r) ; \quad j=1,2, \ldots \ldots .6 \text {, } \\
& M_{6 j}(r)=M_{3 j}(r) ; \quad j=1,2,3,4, \quad M_{65}(r)=0, \quad M_{66}(r)=-H_{1}^{(2)}\left(\xi_{o f} r\right),
\end{aligned}
$$




$$
\begin{array}{lll}
N_{21}(r)=\left(R \delta_{1}^{2}-Q\right) \xi_{1}^{2} J_{1}\left(\xi_{1} r\right), & N_{22}(r)=\left(R \delta_{1}^{2}-Q\right) \xi_{1}^{2} Y_{1}\left(\xi_{1} r\right), & N_{25}(r)=0, \\
N_{23}(r)=\left(R \delta_{2}^{2}-Q\right) \xi_{2}^{2} J_{1}\left(\xi_{2} r\right), & N_{24}(r)=\left(R \delta_{2}^{2}-Q\right) \xi_{2}^{2} Y_{1}\left(\xi_{2} r\right), & N_{26}(r)=0 .
\end{array}
$$

\section{Frequency equation}

For perfect contact of solid and fluids at the interface, the displacements and stresses are continuous. Thus the boundary conditions for a poroelastic circular cylindrical shell immersed in an elastic fluid for a permeable surface are

$$
\begin{aligned}
& \sigma_{r r}+s+P_{i f}=0, \quad s=0, \quad u-u_{i f}=0, \quad \text { at } \quad r=r_{1}, \\
& \sigma_{r r}+s+P_{o f}=0, \quad s=0, \quad u-u_{o f}=0, \quad \text { at } \quad r=r_{2} .
\end{aligned}
$$

The boundary conditions for a poroelastic circular cylindrical shell immersed in an elastic fluid for an impermeable surface are

$$
\begin{aligned}
& \sigma_{r r}+s+P_{i f}=0, \quad \frac{\partial s}{\partial r}=0, \quad u-u_{i f}=0, \quad \text { at } r=r_{1}, \\
& \sigma_{r r}+s+P_{o f}=0, \quad \frac{\partial s}{\partial r}=0, \quad u-u_{o f}=0, \quad \text { at } \quad r=r_{2} .
\end{aligned}
$$

Substitution of equations (14) into equation (16) result in a system of six homogeneous algebraic equations in six constants $C_{1}, C_{2}$, $C_{3}, C_{4}, A_{\text {if }}$, and $A_{\text {of }}$. For a non-trivial solution, the determinant of the coefficients must vanish. By eliminating these constants, the frequency equation of radial vibrations of poroelastic circular cylindrical shell immersed in an acoustic medium, in case of a permeable surface is

$$
\left|A_{i j}\right|=0, \quad i, j=1,2, \ldots \ldots 6 .
$$

In equation (18), the elements $A_{i j}$ are

$$
\begin{array}{ll}
A_{i j}=M_{i j}\left(r_{1}\right), & i=1,2,3 \quad \text { and } \quad j=1,2,3,4,5,6, \\
A_{i j}=M_{i j}\left(r_{2}\right), & i=4,5,6 \quad \text { and } \quad j=1,2,3,4,5,6,
\end{array}
$$

where $M_{i j}(r)$ are defined in equation (15).

Arguing on similar lines, equations (14) and (17) yield the frequency equation of radial vibrations of poroelastic circular cylindrical shell of infinite extent immersed in an acoustic medium, for an impermeable surface to be

$$
\left|B_{i j}\right|=0, \quad i, j=1,2, \ldots \ldots .6 .
$$

where the elements $B_{i j}$ are

$$
\begin{aligned}
& B_{i j}=A_{i j}, \quad i=1,3,4,6 \quad \text { and } \quad j=1,2,3,4,5,6, \\
& B_{i j}=N_{2 j}\left(r_{1}\right), \quad j=1,2,3,4,5,6, \\
& B_{5 j}=N_{2 j}\left(r_{2}\right), \quad j=1,2,3,4,5,6,
\end{aligned}
$$

where $M_{i j}(r)$ and $N_{i j}(r)$ are defined in equation (15).

Equations (18) and (20) are the frequency equations of radial vibrations of poroelastic circular cylindrical shells immersed in an acoustic medium for a permeable and an impermeable surface, respectively. These equations were obtained in the paper studied by Ahmed Shah (2012) as a particular case of axially symmetric vibrations when the wavenumber is vanished. These equations were neither analyzed theoretically nor solved numerically which is warranted. Also there was no graphical representation for the variation of frequency. Moreover, the particular cases, i.e., radial vibrations of fluid-filled poroelastic cylindrical shell and radial vibrations of poroelastic shell surrounded by fluid were not discussed. Therefore, the authors independently solved the problem of radial vibrations of poroelastic cylindrical shell immersed in an acoustic medium. The frequency equation and its particular cases are numerically solved and the obtained frequencies are presented graphically as a function of ratio of thickness to inner radius. Now, we consider different special cases when the outer or the inner fluid vanishes in sections 4(a), 4(b) and 4(c).

\section{4(a) Frequency equation for a fluid-filled poroelastic cylindrical shell}

When the outer fluid density is zero, that is, $\rho_{o f}=0$ then the poroelastic cylindrical shell immersed in an acoustic medium will become a fluid-filled poroelastic cylindrical shell. Thus, the frequency equation of a permeable surface (18) reduce to

$$
\left|A_{i j}\right|=0, \quad i, j=1,2,3,4,5,
$$

where the elements $A_{i j}$ are defined in equation (19) for $\rho_{o f}=0$.

Equation (22) is the frequency equation of radial vibrations of fluid-filled poroelastic cylindrical shell in case of a permeable surface. 
Similarly, the frequency equation of radial vibrations of fluid-filled poroelastic cylindrical shell for an impermeable surface is

$$
\left|B_{i j}\right|=0, \quad i, j=1,2,3,4,5 \text {. }
$$

In equation (23), the elements $B_{i j}$ are defined in equation (21) for $\rho_{o f}=0$.

\section{4(b) Frequency equation for poroelastic cylindrical shell surrounded by fluid}

When the inner fluid vanishes, that is, $\rho_{i f}=0$ then the poroelastic cylindrical shell immersed in an acoustic medium will become a poroelastic cylindrical shell surrounded by fluid. Thus, the frequency equation of a permeable surface (18) reduce to

$$
\left|A_{i j}\right|=0, \quad i=1,2,4,5,6, \quad j=1,2,3,4,6 \text {, }
$$

where the elements $A_{i j}$ are defined in equation (19) for $\rho_{i f}=0$.

Equation (24) is the frequency equation of radial vibrations of poroelastic cylindrical shell surrounded by fluid in case of a permeable surface.

Similarly, the frequency equation of radial vibrations of poroelastic cylindrical shell surrounded by fluid for an impermeable surface is

$$
\left|B_{i j}\right|=0, \quad i=1,2,4,5,6, \quad j=1,2,3,4,6 .
$$

In equation (25), the elements $B_{i j}$ are defined in equation (21) for $\rho_{i f}=0$.

\section{4(c) Frequency equation for poroelastic cylindrical shell in vacuum}

When the inner and outer fluids vanish, that is, $\rho_{i f}=0, \rho_{o f}=0$ then the poroelastic cylindrical shell immersed in an acoustic medium will become a poroelastic cylindrical shell in vacuum. Thus, the frequency equation of a permeable surface (18) reduce to

$$
\left|A_{i j}\right|=0, \quad i=1,2,4,5, \quad j=1,2,3,4,
$$

where the elements $A_{i j}$ are defined in equation (19) for $\rho_{i f}=0$ and $\rho_{o f}=0$. surface is

Similarly, the frequency equation of radial vibrations of poroelastic cylindrical shell in vacuum for an impermeable

$$
\left|B_{i j}\right|=0, \quad i=1,2,4,5, \quad j=1,2,3,4 \text {. }
$$

In equation (27), the elements $B_{i j}$ are defined in equation (21) for $\rho_{i f}=0$ and $\rho_{o f}=0$.

Equations (26) and (27) are the frequency equations of radial vibrations of poroelastic cylindrical shells in vacuum for a permeable and an impermeable surface, respectively, discussed by the Tajuddin and Ahmed Shah (2010).

\section{Non-dimensionalization of frequency equation}

To analyze the frequency equations it is convenient to introduce the following non-dimensional variables:

$$
\begin{aligned}
& a_{1}=P H^{-1}, \quad a_{2}=Q H^{-1}, \quad a_{3}=R H^{-1}, \quad a_{4}=N H^{-1}, \\
& \tilde{x}=\left(V_{0} V_{1}^{-1}\right)^{2}, \quad \tilde{y}=\left(V_{0} V_{2}^{-1}\right)^{2}, \quad m_{11}=\rho_{11} \rho^{-1}, \quad m_{12}=\rho_{12} \rho^{-1}, \quad m_{22}=\rho_{22} \rho^{-1}, \\
& t_{1}=\rho_{i f} \rho^{-1}, \quad t_{2}=\rho_{o f} \rho^{-1}, \quad m_{2}=V_{i f} C_{0}^{-1}, \quad m_{3}=V_{o f} C_{0}^{-1}, \quad \Omega=\omega h C_{0}^{-1},
\end{aligned}
$$

where $\Omega$ is non-dimensional frequency, $H=P+2 Q+R, \rho=\rho_{11}+2 \rho_{12}+\rho_{22}, C_{0}$ and $V_{0}$ are the reference velocities $\left(C_{0}{ }^{2}=N / \rho\right.$, $\left.V_{0}^{2}=H / \rho\right)$, h is the thickness of the poroelastic cylindrical shell. Let

$$
g=\frac{r_{2}}{r_{1}}, \quad \text { so that } \quad \frac{h}{r_{1}}=(g-1) .
$$

\section{Results and discussion}

Two types of poroelastic materials are considered to carry out the computational work, one is sandstone saturated with kerosene, say Material-I (Fatt, 1959). The other one is sandstone saturated with water, Material-II (Yew and Jogi, 1976). Non-dimensional physical parameters of these materials are given in Table- 1 .

Table - 1

\begin{tabular}{|l|l|l|l|l|l|l|l|l|l|}
\hline $\begin{array}{l}\text { Material/ } \\
\text { Parameter }\end{array}$ & $a_{1}$ & $a_{2}$ & $a_{3}$ & $a_{4}$ & $m_{11}$ & $m_{12}$ & $m_{22}$ & $\widetilde{\mathrm{x}}$ & $\widetilde{\mathrm{y}}$ \\
\hline I & 0.843 & 0.065 & 0.028 & 0.234 & 0.901 & -0.001 & 0.101 & 0.999 & 4.763 \\
\hline II & 0.960 & 0.006 & 0.028 & 0.412 & 0.877 & 0 & 0.123 & 0.913 & 4.347 \\
\hline
\end{tabular}


For a given poroelastic material, frequency equations (18) and (20), (22) and (23), (24) and (26) when non-dimensionalized using equations (28) and (29), constitute a relation between non-dimensional frequency $\Omega$ and ratio of thickness to inner radius $\mathrm{h} / \mathrm{r}_{1}$. For poroelastic cylindrical shells immersed in an acoustic medium, the values of $\mathrm{m}_{2}, \mathrm{~m}_{3}, \mathrm{t}_{1}$ and $\mathrm{t}_{2}$ are taken as $\mathrm{m}_{2}=\mathrm{m}_{3}=1.5$ and $t_{1}=t_{2}=0.4$. For broad spectrum of values of $h / r_{1}$, frequency $\Omega$ is computed for the considered poroelastic materials-I and II. The non-dimensional form of the said equations is solved numerically to compute the frequency following the analysis of Gazis (1959). Non-dimensional frequency as a function of ratio of wall-thickness to inner radius each for a permeable and an impermeable surface is presented for poroelastic cylindrical shells immersed in an acoustic medium, fluid-filled poroelastic cylindrical shells and poroelastic shells surrounded by fluid in figures.1-3 respectively. From fig. 1 it is clear that with the increase of thickness there is a gradual increase in the frequency each for a permeable and an impermeable surface beyond $\mathrm{h} / \mathrm{r}_{1}=3.5$ for the considered materials. In general, the frequency for a permeable surface is higher than that of an impermeable surface for Material-I. In case of Material-II, the frequency of an impermeable surface is higher than that of a permeable surface when $\mathrm{h} / \mathrm{r}_{1}$ is less than 5.5. Beyond this, the frequency of a permeable and an impermeable surface is same.

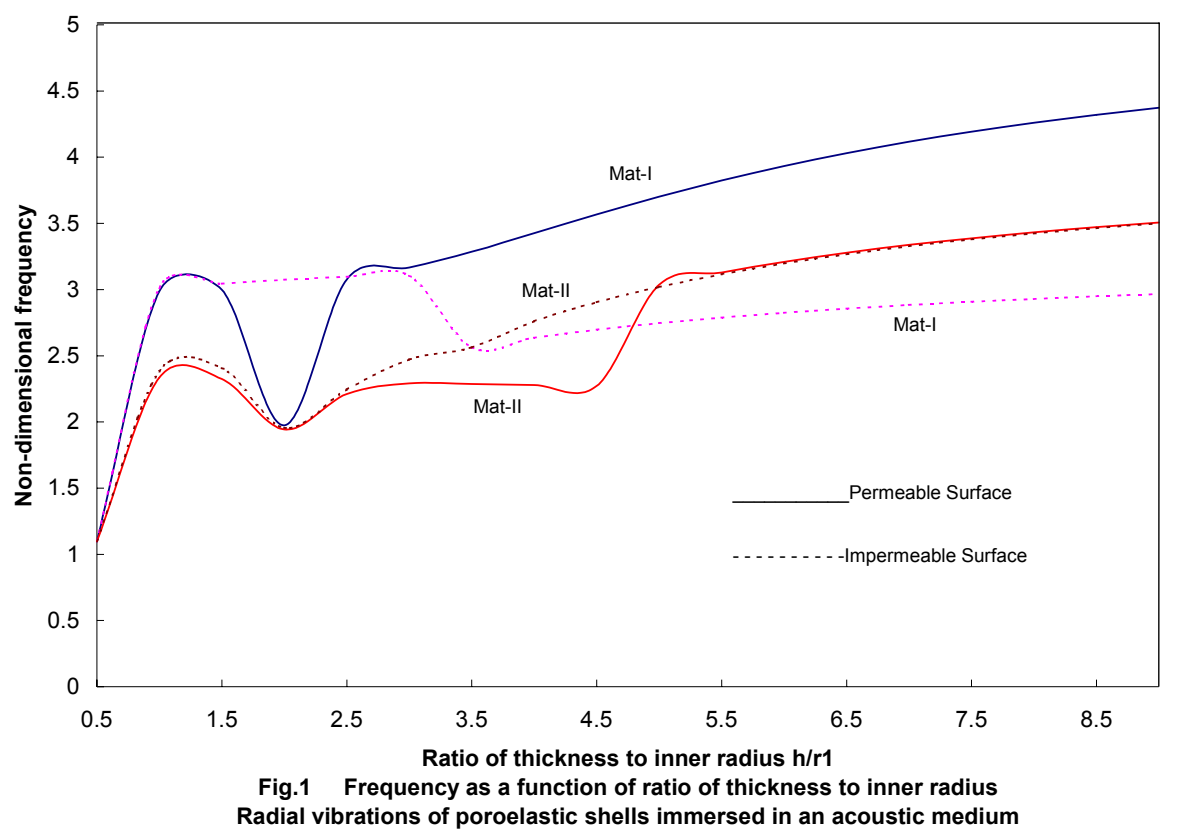

Fig.2 shows the frequency of radial vibrations of fluid-filled poroelastic shells for permeable and impermeable surfaces for the considered materials. Fig. 2 shows that for small thickness there is a decrease of frequency with the increase of $h / r_{1}$. Beyond $\mathrm{h} / \mathrm{r}_{1}=1.5$ there is an increase in the frequency each for a permeable and an impermeable surface. Also it is seen that for small values of $h / r_{1}$, the frequency of permeable and impermeable surfaces is same each for Material-I and Material-II. Moreover, the frequency of Material-II is less than that of Material-I for small values of $h / r_{1}$. Beyond $h / r_{1}=3.5$, the frequency of permeable and impermeable surfaces is almost same in case of Material-II while the frequency of an impermeable surface is higher than that of a permeable surface in case of Material-I.

Fig. 3 shows the frequency as a function of $\mathrm{h} / \mathrm{r}_{1}$ for poroelastic cylindrical shells surrounded by an elastic fluid. From fig. 3 it is clear that the frequency of permeable and impermeable surfaces is same in case of Material-II. The frequency of a permeable surface is higher than that of an impermeable surface in case of Material-I. Also there is a decrease in frequency for small values of $\mathrm{h} / \mathrm{r}_{1}$ as in fig.2. Moreover, with the increase of values of $\mathrm{h} / \mathrm{r}_{1}$ there is a gradual increase in the frequency. In addition the frequency of an impermeable surface for Material-II is higher than that of Material-I. Mainly it is seen that the results tally with the results obtained in the previous papers such as Ahmed Shah (2008). Even though the vibrations considered are different, for example, axially symmetric vibrations of fluid filled shell and in the present case radial vibrations we see that the frequency is higher for an impermeable surface than that of a permeable surface. The numerical results obtained in the paper by Ahmed Shah (2012) are completely different from the results obtained in the present paper. And also the numerical results of paper by Shanker et al (2013b) are obtained for poroelastic fluid-loaded sphere immersed in fluid. 

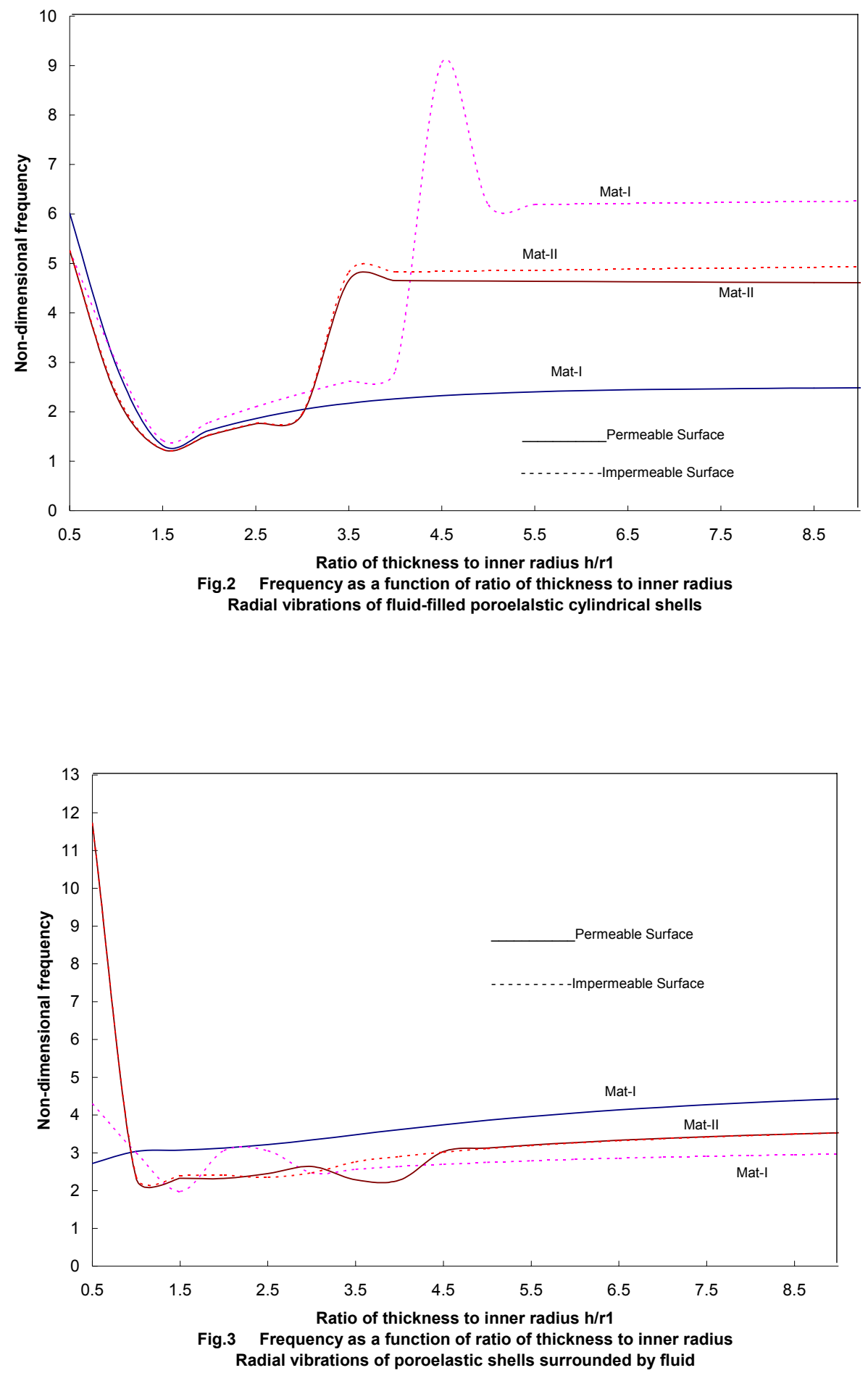

\section{Concluding remarks}

The investigation has lead to the following conclusion:

- The waves propagating in poroelastic cylindrical shells immersed in an acoustic medium, fluid-filled shells and shells surrounded by fluid are highly dispersive when the thickness of shells are small. Also with the increase of thickness of shells, there is a decrease in dispersion.

- In general, it is seen that the frequency of a permeable and an impermeable surface is almost same in case of Material-II while it is not for Material-I. 
- In case of Material-I, it is interesting to note that the frequency of a permeable surface is higher than that of an impermeable surface for poroelastic cylindrical shells immersed in an acoustic medium and shells surrounded by elastic fluid.

- The frequency of radial vibrations of an impermeable surface is higher than that of a permeable surface in case of fluidfilled poroelastic cylindrical shells.

\section{Nomenclature}

$\begin{array}{ll}(r, \theta, z) & \text { Cylindrical polar coordinates } \\ \rho_{11}, \rho_{12}, \rho_{22} & \text { Mass coefficients } \\ \vec{u} & \text { Solid displacement } \\ \vec{U} & \text { Liquid displacement } \\ m_{11}, m_{12}, m_{22} & \text { Non-dimensional mass coefficients } \\ r_{2}, r_{1} & \text { Outer radius, inner radius respectively } \\ b & \text { Dissipation } \\ A, N, Q, R & \text { Poroelastic constants } \\ \omega & \text { Circular frequency } \\ \Omega & \text { Non-dimensional frequency } \\ V_{1}, V_{2} & \text { Dilatational wave velocities of find and second kind } \\ C_{0}, V_{0} & \text { Reference velocities } \\ e & \text { Dilatation of solid } \\ \in & \text { Dilatation of liquid } \\ \sigma_{i j} & \text { Stresses } \\ S & \text { Liquid pressure } \\ H_{\mathrm{n}}^{(2)} & \text { Hankel function of second kind of order } n \\ h & \text { Thickness of the hollow poroelastic cylinder } \\ g & \text { Ration of outer to inner radius } \\ J_{n}, Y_{n} & \text { Bessel functions of first and second kind of order } n\end{array}$

\section{Acknowledgement}

Authors are thankful to the reviewers for their useful suggestions.

\section{References}

Ahmed Shah, S., 2008. Axially symmetric vibrations of fluid-filled poroelastic circular cylindrical shells. Journal of Sound and Vibration. Vol.318, pp.389-405.

Ahmed Shah, S., 2012. Vibration analysis of an infinite poroelastic circular cylindrical shell immersed in fluid. Open Journal of Acoustics. Vol. 2, pp. 86-93.

Ahmed Shah, S., and Tajuddin, M., 2009. Axially symmetric vibrations of finite composite poroelastic cylinders. International Journal of Applied Mechanics and Engineering. Vol.14, pp. 865-877.

Ahmed Shah, S., and Tajuddin, M., 2010. On flexural vibrations of poroelastic circular cylindrical shells immersed in an acoustic medium. Special Topics and Reviews in Porous Media - An International Journal. Vol.1, pp.67-78.

Ahmed Shah, S., 2011. Flexural wave propagation in coated poroelastic cylinders with reference to fretting fatigue. Journal of Vibration and Control. Vol.17, pp.1049-1064.

Ahmed Shah, S., and Tajuddin, M., 2011. On axially symmetric vibrations of fluid filled poroelastic spherical shells. Open Journal of Acoustics. Vol.1, pp.15-26.

Biot, M.A., 1956. Theory of propagation of elastic waves in fluid-saturated porous solid. Journal of the Acoustical Society of America. Vol.28, pp.168-178.

Chao, G., Smeulders, D.M.J., and van Dongen, M.E.H., 2004. Shock-induced borehole waves in porous formations: Theory and experiments. Journal of the Acoustical Society of America. Vol.116, pp. 693-702.

Fatt, I., 1959. The Biot-Willis elastic coefficients for a sandstone. Journal of Applied Mechanics. Vol.26, pp.296-297. 
Gazis, D.C., 1959. Three-dimensional investigation of the propagation of waves in hollow circular cylinders. I., Analytical foundation. Journal of the Acoustical Society of America. Vol.31, pp.568-578.

Marilyn J. Berliner and Solecki, R., 1996 Wave propagation in fluid-loaded transversely isotropic cylinders. Part I - Analytical formulation. Journal of the Acoustical Society of America. Vol.99, pp.1841-1847.

McFadden, J.A., 1954. Radial vibrations of thick-walled hollow cylinders. Journal of the Acoustical Society of America. Vol.26, pp.714-715.

Shanker, B., Nageswara Nath, C., Ahmed Shah, S., and Manoj Kumar, J., 2013a. Vibration analysis of poroelastic composite hollow sphere. Acta Mechanica. Vol.224, pp.327-341.

Shanker, B., Nageswara Nath, C., Ahmed Shah, S., and Manoj Kumar, J., 2013b. Free vibrations of fluid-loaded poroelastic hollow sphere surrounded by a fluid. International Journal of Applied Mathematics and Mechanics. Vol.9, pp.14-34.

Tajuddin, M., and Ahmed Shah, S., 2010. Radial vibrations of thick-walled hollow poroelastic cylinders. Journal of Porous Media. Vol.13, pp.307-318.

Wisse, C.J., Smeulders, D.M.J., van Dongen, M.E.H., and Chao, G., 2002. Guided wave modes in porous cylinders: Experimental results. Journal of the Acoustical Society of America. Vol.112, pp.890-895.

Yew, C.H., and Jogi, P.N., 1976. Study of wave motions in fluid-saturated porous rocks. Journal of the Acoustical Society of America. Vol.60, pp.2-8.

\section{Biographical notes}

M. Ramesh is working as Assistant Professor (C) in the Department of Mathematics, University College of Technology, Osmania University, Hyderabad.

Dr. S. Ahmed Shah is Professor of Mathematics in Deccan College of Engineering and Technology, Hyderabad.

Dr. M. V. Ramana Murthy is Professor in the Department of Mathematics, Osmania University, Hyderabad.

Received March 2014

Accepted June 2014

Final acceptance in revised form July 2014 